\title{
Existence and Uniqueness of Isothermal, Slightly Compressible Stratified Flow
}

\author{
Arianna Passerini®D \\ Communicated by G. P. Galdi
}

\begin{abstract}
We show well-posedness for the equations describing a new model of slightly compressible fluids. This model was recently rigorously derived in Grandi and Passerini (Geophys Astrophys Fluid Dyn, 2020) from the full set of balance laws and falls in the category of anelastic Navier-Stokes fluids. In particular, we prove existence and uniqueness of global regular solutions in the two-dimensional case for initial data of arbitrary "size", and for "small" data in three dimensions. We also show global stability of the rest state in the class of weak solutions.
\end{abstract}

Mathematics Subject Classification. 76E06, 76E19, 76B03.

Keywords. Existence, Uniqueness, Stability compressible fluids, Anelastic approximation.

\section{Introduction}

The Oberbeck-Boussinesq approximation $[1,20]$ is a very popular model, used to describe convection in a horizontal layer of fluid heated from below [14]. As is well known, the basic assumption is that the fluid is incompressible, namely, the velocity field $\mathbf{v}$ satisfies $\nabla \cdot \mathbf{v}=0$ at all points and times, whereas the density depends solely on the temperature $T$, and its contribution becomes relevant only in the buoyancy term.

However, as noticed for instance in $[10,11,18,21]$, thermodynamic variables such as energy and density cannot be a function of $T$ only, but should also depend on the pressure $p$ since, otherwise, Gibbs law would be unattended and stability in waves propagation not allowed. It turns out that, for these more general models, one has necessarily to relax the solenoidal condition on $\mathbf{v}[6,17]$ to enlarge the region of the non-dimensional parameter space in which formal limits lead to reliable approximations for compressible fluids.

Motivated by this important issues, the author jointly with D. Grandi rigorously derived, by perturbative methods from the full set of balance laws, new models where the density, $\rho$, may depend on both $T$ and $p[12,13]$, while for related ones, the authors addressed well-posedness and stability questions, in $[2,4,5]$. In particular, in [13], we proposed a new model for thermal convection in a horizontal layer of fluid heated from below with $\rho=\rho(T, p)$. In such a case, the original full compressible system allows for an elementary solution $\mathrm{s}_{e}:=\left(T_{e}, p_{e}\right)$ with corresponding $\rho_{e}=\rho\left(T_{e}, p_{e}\right)$; see $(2.2),(2.4)$, and (2.5). The relevant equation are then obtained from the full system by a perturbation expansions around $\mathrm{s}_{e}$, by using as non-dimensional small parameter $\alpha \delta T$, where $\alpha$ is the thermal expansion coefficient and $\delta T$ is the temperature difference between the two horizontal planes confining the fluid. The limiting equations thus derived $\left(\mathrm{see}(\mathrm{OB})_{\beta}\right)$ fall, in the isothermal case, in the category of the so called anelastic approximations of the Navier-Stokes equations; see (2.9), where the velocity field is no longer solenoidal but, instead, satisfies $\nabla \cdot(\rho \mathbf{v})=0$. It is just the latter that makes the new model interesting from the point of view of well-posedness of the corresponding initial-boundary value problem, which constitutes the focus of this article. 
In this regard, in the recent paper [15] the authors investigate tha above questions for a general class of anelastic models. However, they assume, among other things, that the density can be extended to the whole space to a smooth periodic function in the coordinate orthogonal to the layer. Unfortunately, this assumption is not satisfied in our case, since $\rho_{e}$ assumes different values on the planes confining the layer; see $(2.5)$.

As a result, we use a different strategy that, in our opinion, is the natural extension of classical methods used for the Navier-Stokes equations, at least when, as in the case at hand, the density is strictly positive. More precisely, we study our problem in the functional framework where

$$
\nabla \cdot\left(\rho_{e} \mathbf{v}\right)=0 .
$$

The crucial points are the proof of a Helmholtz-like decomposition of the Lebesgue space $L^{2}$ (Lemma 4.2) and maximal $L^{2}$-regularity for a Stokes-like operator (Lemma 4.3) where, in both cases, the classical solenoidality condition on $\mathbf{v}$ is replaced by the request (1). With thse results in hand, we can then suitably modify the standard Galerkin method as employed in [19] and show different existence and uniqueness results. Precisely, under the assumption of stress-free boundary condition and periodicity in the horizontal coordinates, we first prove existence of weak solutions in both two- and three-dimensional cases (Theorem 3.1). Successively, if the initial data are more regular but arbitrary in "size", we prove existence and uniqueness of strong solutions (in the sense of Prodi) in two dimensions (Theorem 3.2). However, in dimension three, as expected, the same conclusion holds only for "small" time intervals or for arbitrary times, but "small" initial data (Theorem 3.3).

The plan of the paper is as follows. After formulating the problem in Sect. 2, in the following Sect. 3 we give the definition of weak solution and state the existence and uniqueness theorems. In Sect. 4, we prove a Helmholtz-like decomposition involving vector fields satisfying (1) and introduce a Stokes-like operator for which we prove maximal $L^{2}$ regularity. In the remaining two sections we give a proof of our theorems: for weak solutions, in Sect. 5, and strong solutions, in Sect. 6.

\section{Formulation of the Problem}

Assume the fluid occupies a horizontal layer comprised between the unmovable planes placed at $z=0$ and $z=h$, subject to the gravity force $\rho \mathbf{g}$. The planes are kept at constant, not necessarily equal, temperatures namely, $T=T_{d}$ at $z=0$ and $T=T_{d}-\delta T, \delta T \in \mathbb{R}$, at $z=h$. Under isothermal conditions $\delta T=0$, the constitutive equation $\rho=\rho_{0}=$ constant allows for the fluid to be at rest $(\mathbf{v} \equiv 0)$ with corresponding hydrostatic pressure $p=-\rho g z$. On the other hand, if $\delta T \neq 0$, then the fluid is still at rest while the "classical" Boussinesq constitutive equation

$$
\rho=\rho_{d}\left[1-\alpha\left(T-T_{d}\right)\right],
$$

implies the well-known linear profile for the temperature

$$
T_{e}(z)=T_{d}-\frac{\delta T}{h} z .
$$

The latter, in turn, furnishes the following expression for the pressure field [1]

$$
p_{e}(z)=p_{d}-\rho_{d} g\left(z+\frac{\alpha \delta T}{2 h} z^{2}\right) .
$$

In [18], the author jointly with T. RUGGERI have introduced a constitutive equation more general than (2.1) to include also pressure variations:

$$
\rho=\rho_{d}\left[1-\alpha\left(T-T_{d}\right)+\beta\left(p-p_{d}\right)\right], \quad \beta \neq 0 .
$$

In such a case, while the stratified temperature field remains unchanged, the pressure distribution becomes [18]:

$$
p_{e}(z)=p_{d}+\frac{e^{-\rho_{d} g \beta z}-1}{\beta}+\frac{\alpha \delta T}{\beta}\left(\frac{1-e^{-\rho_{d} g \beta z}}{\rho_{d} g h \beta}-\frac{z}{h}\right) .
$$


Notice that in the isothermal case $\delta T=0$, combining (2.4) and (2.3), we find

$$
\rho_{e}=\rho_{d} e^{-\beta \rho_{d} g z},
$$

which provides the compatible stratification of the fluid.

The main accomplishment of [13] was to derive, as formal limit from the full compressible model, an approximate set of equations for the perturbation $(\tau, P, \mathbf{v})$ to the basic solution $\left(T_{e}, p_{e}, \mathbf{v} \equiv 0\right)$, that generalizes the classical O-B system by taking into account the compressibility of the fluid (anelastic O-B system). More precisely, setting

$$
\tau:=T-T_{e}, \quad P:=p-p_{e}, \quad \mathbf{v}=\left(v^{x}, v^{y}, v^{z}\right):=\mathbf{v}-0,
$$

in [13] it is shown that $(\tau, P, \mathbf{v})$ must satisfy the following nondimensional equations ${ }^{1}$

$$
(\mathrm{OB})_{\beta} \quad\left\{\begin{array}{l}
\nabla \cdot \mathbf{v}=\beta v^{z}, \\
e^{-\beta z}\left(\mathbf{v}_{t}+\mathbf{v} \cdot \nabla \mathbf{v}\right)-\operatorname{Pr}\left(\beta \gamma \nabla v^{z}+\Delta \mathbf{v}\right)=-\nabla P+(\sqrt{\operatorname{Ra}} \operatorname{Pr} \tau-\beta P) \mathbf{k}, \\
e^{-\beta z}\left(\tau_{t}+\mathbf{v} \cdot \nabla \tau+\xi \sqrt{\operatorname{Ra}} v^{z}\right)-\Delta \tau=0,
\end{array}\right.
$$

where $\tau, P, \mathbf{v}$ and $\beta$ are meant now to be nondimensional quantities. Moreover,

$$
\operatorname{Pr}:=\frac{\mu}{\rho_{d} \kappa}, \quad \operatorname{Ra}:=\frac{\alpha|\delta T| \rho_{d} g h^{3}}{\mu \kappa}, \quad \gamma:=\frac{\zeta}{\mu}+\frac{1}{3},
$$

with $\mu, \zeta$ and $\kappa$ being, respectively, shear and bulk viscosities and thermal conductivity of the fluid. Finally, $\xi= \pm 1$, according to whether $\delta T \lessgtr 0$.

In what follows, we are interested in the isothermal case $\delta T=0$ (i.e. Ra $=0$ ), in which case, by $(2.2)$ and (2.3), the basic state reduces to

$$
T_{e}=T_{d}, \quad p_{e}(z)=p_{d}+\frac{e^{-\rho_{d} g \beta z}-1}{\beta}, \quad \mathbf{v} \equiv 0,
$$

while $(\mathrm{OB})_{\beta}$ decouples into the following two sets of equations

$$
\left\{\begin{array}{l}
\nabla \cdot \mathbf{v}=\beta v^{z} \\
e^{-\beta z}\left(\mathbf{v}_{t}+\mathbf{v} \cdot \nabla \mathbf{v}\right)-\operatorname{Pr}\left(\beta \gamma \nabla v^{z}+\Delta \mathbf{v}\right)=-\nabla P-\beta P \mathbf{k} .
\end{array}\right.
$$

and

$$
e^{-\beta z}\left(\tau_{t}+\mathbf{v} \cdot \nabla \tau\right)-\Delta \tau=0
$$

Introducing the new variable

$$
\Pi:=e^{\beta z}\left(\frac{1}{\operatorname{Pr}} P-\gamma \beta v^{z}\right),
$$

it is easy to see that $(2.7)$ can be rewritten as follows

$$
\left\{\begin{array}{l}
\nabla \cdot\left(e^{-\beta z} \mathbf{v}\right)=0 \\
\frac{1}{\operatorname{Pr}}\left(\mathbf{v}_{t}+\mathbf{v} \cdot \nabla \mathbf{v}\right)-e^{\beta z} \Delta \mathbf{v}=-\nabla \Pi-e^{\beta z} \gamma \beta^{2} v^{z} \mathbf{k}
\end{array}\right.
$$

We shall study (2.9) in the space-time domain $\Omega_{0} \times(0, \infty)$, where

$$
\Omega_{0}=\left\{\left(x^{\prime}, z\right) \in \mathbb{R}^{n}: x^{\prime} \in \mathbb{T}^{n-1}, z \in(0,1)\right\}, \quad n=2,3,
$$

and $x^{\prime}=(x, y)$ if $n=3$ and $x^{\prime}=x$ if $n=2$. Moreover, we shall adopt stress-free boundary condition, namely,

$$
v^{z}=0, \quad \partial_{z} v^{x^{\prime}}=0 \text { at } z=0,1,
$$

where $v^{x^{\prime}}=\left(v^{x}, v^{y}\right)$ if $n=3$ and $v^{x^{\prime}}=v^{x}$, if $n=2$. Finally, to exclude rigid motions, we assume that the mean value of $v^{x^{\prime}}$ is zero at all times:

$$
\int_{\Omega_{0}} v^{x^{\prime}}\left(x^{\prime}, z, t\right) d V=0
$$

\footnotetext{
${ }^{1}$ We shall use the following notation: $(\cdot)_{t} \equiv \partial(\cdot) / \partial t$, and $\partial_{\xi}(\cdot) \equiv \partial(\cdot) / \partial \xi$, where $\xi$ denotes any spatial variable.
} 
Before stating our main results, we need to introduce some notation. By $L^{q}\left(\Omega_{0}\right)$ and $W^{m, q}\left(\Omega_{0}\right)$ $q \in[1, \infty], m \in \mathbb{N}$, we denote the usual Lebesgue and Sobolev spaces with associated norm $\|\cdot\|_{q}$ and $\|\cdot\|_{m, q}$, respectively. If $q=2$, we indicate the associated scalar product in $L^{2}$ by $(\cdot, \cdot) . \hat{\mathcal{L}}^{2}\left(\Omega_{0}\right)$ stands for the subspace of $L^{2}\left(\Omega_{0}\right)$ of those vector fields $\mathbf{v}$ satisfying $(2.10)_{1}$ and $(2.9)_{1}$ in a weak form, namely,

$$
\left(e^{-\beta z} \mathbf{v}, \nabla \varphi\right)=0, \text { for all } \varphi \in W^{1,2}\left(\Omega_{0}\right) .
$$

Furthermore, by $\hat{W}^{2, q}\left(\Omega_{0}\right)$ we denote the subspace of $W^{2, q}\left(\Omega_{0}\right)$ of functions satisfying (2.10), and by $\hat{W}^{1,2}\left(\Omega_{0}\right)$ the subspace of $W^{1,2}\left(\Omega_{0}\right)$ of functions satisfying $(2.10)_{1}$. We also set $\hat{\mathcal{W}}^{1,2}\left(\Omega_{0}\right):=\hat{\mathcal{L}}^{2}\left(\Omega_{0}\right) \cap$ $\hat{W}^{1,2}\left(\Omega_{0}\right)$, and denote by $\hat{\mathcal{W}}^{-1,2}\left(\Omega_{0}\right)$ its dual, with corresponding norm $\|\cdot\|_{-1,2}$. Finally, for $r \in[1, \infty]$, let $L^{r}\left(0, T ; W^{m, q}\left(\Omega_{0}\right)\right), T>0$, be the space of functions $\mathbf{w}$ such that

$$
|\mathbf{w}|_{r, m, q}:=\left\{\begin{array}{l}
\left(\int_{0}^{T}\|\mathbf{w}(t)\|_{m, q}^{r} d t\right)^{\frac{1}{r}}<\infty, \quad \text { if } r \in[1, \infty) \\
\operatorname{ess} \sup _{t \in[0, T]}\|\mathbf{w}(t)\|_{m, q}<\infty, \quad \text { if } r=\infty
\end{array}\right.
$$

The subscript $m$ is omitted in case $m=0$.

\section{Statement of the Main Results}

Our main goal is the investigation of the well posedeness of the initial-boundary value problem associated to (2.9). As in the case of the classical Navier-Stokes model, the result may depend on the Euclidean dimension. We begin to give the definition of weak solution.

Definition 3.1. A vector field $\mathbf{v} \in L^{\infty}\left(0, T ; \hat{\mathcal{L}}^{2}\left(\Omega_{0}\right)\right) \cap L^{2}\left(0, T ; W^{1,2}\left(\Omega_{0}\right)\right)$ is a weak solution to the problem (2.9)-(2.10) corresponding to the initial data $\mathbf{v}^{0} \in \hat{\mathcal{L}}^{2}\left(\Omega_{0}\right)$ if, for all $\boldsymbol{\Psi} \in \hat{\mathcal{W}}^{1,2}\left(\Omega_{0}\right)$ and all $t \in(0, T)$ it satisfies the following integral equation

$$
\frac{1}{\operatorname{Pr}}\left(e^{-\beta z} \mathbf{v}(t), \boldsymbol{\Psi}\right)+\int_{0}^{t}\left[(\nabla \mathbf{v}(s), \nabla \boldsymbol{\Psi})+\left(\frac{1}{\operatorname{Pr}} e^{-\beta z} \mathbf{v} \cdot \nabla \mathbf{v}(s)+\beta^{2} \gamma v^{z}(s), \Psi\right)\right] d s=\frac{1}{\operatorname{Pr}}\left(e^{-\beta z} \mathbf{v}^{0}, \Psi\right) .
$$

Formally, (3.1) is obtained by multiplying $(2.9)_{2}$ with $e^{-\beta z} \boldsymbol{\Psi}$, integrating by parts over $\Omega_{0} \times(0, t)$ and using $(2.9)_{1}$.

We now collect the main results proved in this paper in the form of as many theorems. We begin with the following one.

Theorem 3.1. Let $n=2,3$ and let $\operatorname{Pr}, \gamma$ and $\beta$ be given. Then, for any initial data $\mathbf{v}^{0} \in \hat{\mathcal{L}}^{2}\left(\Omega_{0}\right)$, there exists at least one weak solution for all $T>0$. Moreover, such a solution satisfies the following decay property

$$
\|\mathbf{v}(t)\|_{2, \beta} \leq\left\|\mathbf{v}^{0}\right\|_{2, \beta} e^{-\gamma_{P} \operatorname{Pr} t}, \quad t \geq 0 .
$$

We also prove existence and uniqueness of more regular solutions, in the form stated in the following theorems.

Theorem 3.2. Let $\Omega_{0} \subset \mathbb{R}^{2}$. Then, given arbitrary positive $\operatorname{Pr}, \gamma$ and $\beta$, and arbitrary initial data in $\hat{\mathcal{W}}^{1,2}\left(\Omega_{0}\right)$, there exists a unique corresponding weak solution $\mathbf{v}$ to $(2.7)$ which, in addition, is in the class $C\left([0, \infty) ; W^{1,2}\left(\Omega_{0}\right)\right) \cap L^{2}\left((0, \infty) ; W^{2,2}\left(\Omega_{0}\right)\right)$ with $\mathbf{v}_{t} \in L^{2}\left((0, \infty) ; L^{2}\left(\Omega_{0}\right)\right)$. Moreover, there is $\Pi \in$ $L^{2}\left(0, \infty ; L^{2}\left(\Omega_{0}\right)\right)$ with $\nabla \Pi \in L^{2}\left(0, \infty ; L^{2}\left(\Omega_{0}\right)\right)$, such that $(\mathbf{v}, \Pi)$ satisfies $(2.7)$ for a.a. $(x, t) \in \Omega_{0} \times(0, \infty)$.

Theorem 3.3. Let $\Omega_{0} \subset \mathbb{R}^{3}$ and let $\operatorname{Pr}, \gamma, \beta$ be arbitrary positive numbers. Then, for all $\left\|v^{0}\right\|_{1,2}<$ $\infty$ there exist $a T>0$ and a unique corresponding weak solution $\mathbf{v}$ to (2.7) which, in addition, is in the class $C\left([0, T) ; W^{1,2}\left(\Omega_{0}\right)\right) \cap L^{2}\left((0, T) ; W^{2,2}\left(\Omega_{0}\right)\right)$ with $\mathbf{v}_{t} \in L^{2}\left((0, T) ; L^{2}\left(\Omega_{0}\right)\right)$. Moreover, there is $\Pi \in L^{2}\left(0, T ; L^{2}\left(\Omega_{0}\right)\right)$ with $\nabla \Pi \in L^{2}\left(0, T ; L^{2}\left(\Omega_{0}\right)\right)$, such that $(\mathbf{v}, \Pi)$ satisfies $(2.7)$ for a.a. $(x, t) \in$ 
$\Omega_{0} \times(0, T)$. Finally, there exists a constant $C_{0}$ depending only on the above physical parameters, such that if $\left\|\mathbf{v}^{0}\right\|_{2}^{2}\left\|\nabla \mathbf{v}^{0}\right\|_{2}^{2} \leq 2 / C_{0}$ we can take $T=\infty$.

Remark 3.1. The proofs of existence are based on the Galerkin method, in the way suggested by Prodi [19]. However, Prodi's approach must be suitably modified, in that, in our case, the velocity field is no longer solenoidal. This requires the study of full regularity of solutions to a new Stokes problem derived in connection with problem (2.9); see (4.11). The latter, in turn, requires the study of an appropriate Helmholtz-like decomposition of the type $L^{2}\left(\Omega_{0}\right)=\widehat{\mathcal{L}}^{2}\left(\Omega_{0}\right) \oplus G_{\beta}\left(\Omega_{0}\right)$, dictated by the the non-solenoidality of the velocity field (see (4.9)). All these results are contained in Sect. 3, which, in fact, constitutes the heart of the matter of the paper.

Remark 3.2. In the case of three-dimensional weak solutions (Theorem 3.1), following [3] and [16], one can proves a result of strong convergence of the Galerkin approximation (the first known for weak solutions) in $L^{q}\left(0, T ; W^{1,2}\left(\Omega_{0}\right)\right)$ for all $q \in[1,2)$. Actually, in [3] it is stated that such a convergence occurs along the Galerkin approximation with aspecial base. For the sake of the brevity we do not give the details. The interest of this convergence consists in the fact that one can show a sort of energy equality, or equivalently, an evaluation of the possible gap in the inequality see [3].

\section{Preliminary Results}

We start by proving some formal properties of solutions to (2.9)-(2.10). To this end, we observe that, in view of $(2.9)_{1}$, the vector field $\mathbf{u}:=e^{-\beta z} \mathbf{v}$ is solenoidal, in fact

$$
\nabla \cdot \mathbf{u}=-\beta e^{-\beta z} \mathbf{k} \cdot \mathbf{v}+e^{-\beta z} \nabla \cdot \mathbf{v}=0 .
$$

As a consequence, since the factor $e^{-\beta z}$ can be associated to the first term of the bilinear form as well, by integrating by parts and using (2.10) and (4.1), one shows that

$$
\int_{\Omega_{0}} \mathbf{v} \cdot \nabla \mathbf{v} \cdot \mathbf{u} d V=\int_{\Omega_{0}} \mathbf{u} \cdot \nabla \mathbf{v} \cdot \mathbf{v} d V=0
$$

Set

Since

$$
\|\mathbf{v}\|_{2, \beta}:=\left(\int_{\Omega_{0}} e^{-\beta z}|\mathbf{v}|^{2} d V\right)^{\frac{1}{2}}
$$

$$
\int_{\Omega_{0}} e^{-\beta z}|\mathbf{v}|^{2} d V \leq \int_{\Omega_{0}}|\mathbf{v}|^{2} d V \leq e^{\beta} \int_{\Omega_{0}} e^{-\beta z}|\mathbf{v}|^{2} d V
$$

the norms $\|\mathbf{v}\|_{2, \beta}$ and $\|\mathbf{v}\|_{2}$ are equivalent. Property (4.3) will be used throughout, even without explicitly mentioning it. Now, if we formally dot-multiply both sides of $(2.9)_{1}$ by the solenoidal field $\mathbf{u}$, integrate by parts over $\Omega_{0}$ and use (4.2) and (2.10), we deduce the following important relation, for all $\beta \geq 0$

$$
\frac{1}{2 \operatorname{Pr}} \frac{d}{d t}\|\mathbf{v}\|_{2, \beta}^{2}+\|\nabla \mathbf{v}\|_{2}^{2}+\gamma \beta^{2}\left\|v^{z}\right\|_{2}^{2}=0 .
$$

We also notice that, using (4.1) and assuming, without loss that the (constant) equal temperatures on the bounding planes are 0 , (formally) multiplying both sides of (2.8) by $\tau$ and integrating over $\Omega_{0}$, we get

$$
\frac{1}{2} \frac{d}{d t}\|\tau\|_{2}^{2}=-\|\nabla \tau\|_{2}^{2} .
$$

Employing on the right hand side of this equation the scalar version of (4.25) in conjunction with Gronwall's lemma, we obtain, as expected, an exponential decay to the boundary temperature.

Now, we derive some results that will play an important role for the existence results developed in the next section. 
We begin to prove the unique solvability of the following Neumann problem:

$$
\Delta q-\alpha \partial_{z} q=F, \text { in } \Omega_{0}, \quad \partial_{z} q\left(x^{\prime}, 0\right)=\partial_{z} q\left(x^{\prime}, 1\right)=0, \quad \alpha \in \mathbb{R},
$$

in a suitable function class. In order to reach this goal, we observe that (4.5) is (formally) equivalent to the following one

$$
\nabla \cdot\left(e^{-\alpha z} \nabla q\right)=e^{-\alpha z} F:=G, \text { in } \Omega_{0}, \quad \partial_{z} q\left(x^{\prime}, 0\right)=\partial_{z} q\left(x^{\prime}, 1\right)=0,
$$

Let

$$
H:=\left\{q \in W^{1,2}\left(\Omega_{0}\right):(q, 1)=0\right\} .
$$

In view of Wirtinger inequality, $H$ becomes a Hilbert space with respect to the norm induced by the scalar product

$$
\left(e^{-\alpha z} \nabla q_{1}, \nabla q_{2}\right), \quad q_{i} \in H .
$$

We shall say that $q \in H$ is a weak solution to (4.6) if

$$
\left(e^{-\alpha z} \nabla q, \nabla \varphi\right)=-(G, \varphi), \text { for all } \varphi \in H .
$$

Let us denote by $H^{-1}$ the dual space of $H$. We show the following lemma that in the case $\alpha<2 \pi$ was proved in $[2,4]$ by different arguments.

Lemma 4.1. Let $\alpha \in \mathbb{R}$. For any $G \in H^{-1}$, problem (4.6) admits one and only one corresponding weak solution $q$. Moreover, if $G \in L^{2}\left(\Omega_{0}\right)$, then $q \in W^{2,2}\left(\Omega_{0}\right)$ and satisfies (4.5). Finally, there exists $C=C(\alpha)$ such that

$$
\|q\|_{2,2} \leq C\|F\|_{2} .
$$

Proof. Existence of a unique weak solution $q$ is an immediate consequence of the assumption on $G$ and of Riesz representation theorem. Moreover, setting $\phi \equiv q$ in (4.7) we deduce the existence of a positive constant $C$ such that

$$
\|q\|_{1,2} \leq C\|G\|_{H^{-1}} .
$$

Next, assume $G \in L^{2}\left(\Omega_{0}\right)$ and set $\psi:=e^{-\alpha z} \varphi$. From (4.7) we thus get

$$
(\nabla q, \nabla \psi)=(M, \psi), \quad M:=-\alpha \partial_{z} q+F .
$$

Since $F \in L^{2}\left(\Omega_{0}\right)$, by classical elliptic regularity we get that $q \in W^{2,2}\left(\Omega_{0}\right)$, and that it satisfies (4.5) along with $\|q\|_{2,2} \leq C\|M\|_{2}$. The latter, combined with (4.8) completes the proof of the lemma.

An important consequence of the previous result is given in the following lemma.

Lemma 4.2. Let $\beta \in \mathbb{R}$. Then, the space $L^{2}\left(\Omega_{0}\right)$ admits the following orthogonal decomposition

$$
L^{2}\left(\Omega_{0}\right)=\hat{\mathcal{L}}^{2}\left(\Omega_{0}\right) \oplus G_{\beta}\left(\Omega_{0}\right)
$$

where

$$
G_{\beta}\left(\Omega_{0}\right):=\left\{\mathbf{h} \in L^{2}\left(\Omega_{0}\right): \mathbf{h}=e^{-\beta z} \nabla Q, Q \in W^{1,2}\left(\Omega_{0}\right)\right\} .
$$

Proof. By $(2.11) \hat{\mathcal{L}}^{2}\left(\Omega_{0}\right)$ and $G_{\beta}\left(\Omega_{0}\right)$ are orthogonal. For a given $\mathbf{w} \in L^{2}\left(\Omega_{0}\right)$ consider the problem of finding $Q \in H$ such that

$$
\left(e^{-\beta z} \mathbf{w}, \nabla \psi\right)=\left(e^{-2 \beta z} \nabla Q, \nabla \psi\right), \text { for all } \psi \in H .
$$

Since the left hand side defines a bounded linear functional in $H$, the existence of a unique $Q \in H$ is guaranteed by Lemma 4.1. Therefore, setting $\mathbf{v}:=\mathbf{w}-e^{-\beta z} \nabla Q$, we at once show that $\mathbf{v} \in \hat{\mathcal{L}}^{2}\left(\Omega_{0}\right)$. The lemma is thus proved. 
Let

$$
P: L^{2}\left(\Omega_{0}\right) \rightarrow \hat{\mathcal{L}}^{2}\left(\Omega_{0}\right)
$$

be the orthogonal projection operator defined by Lemma 4.2, and introduce the operator

$$
A: \mathbf{v} \in \hat{\mathcal{L}}^{2}\left(\Omega_{0}\right) \cap \hat{W}^{2,2}\left(\Omega_{0}\right) \subset \hat{\mathcal{L}}^{2}\left(\Omega_{0}\right) \mapsto P \Delta \mathbf{v} \in \hat{\mathcal{L}}^{2}\left(\Omega_{0}\right) .
$$

The following result holds.

Lemma 4.3. For any $\boldsymbol{f} \in L^{2}\left(\Omega_{0}\right)$ there exist unique $\mathbf{v} \in W^{2,2}\left(\Omega_{0}\right)$ and $Q \in W^{1,2}\left(\Omega_{0}\right)$ with $(Q, 1)=0$ such that

$$
\begin{aligned}
& \left.\begin{array}{l}
\Delta \mathbf{v}=e^{-\beta z} \nabla Q+\boldsymbol{f} \\
\nabla \cdot\left(e^{-\beta z} \mathbf{v}\right)=0
\end{array}\right\} \quad \text { in } \Omega_{0} \\
& \partial_{z} v^{x^{\prime}}=v^{z}=0 \quad \text { at } z=0,1 .
\end{aligned}
$$

Moreover,

$$
\|\mathbf{v}\|_{2,2}+\|Q\|_{1,2} \leq C\|\boldsymbol{f}\|_{2}
$$

Thus, in particular,

$$
\|\Delta \mathbf{v}\|_{2} \leq C\|P \Delta \mathbf{v}\|_{2} .
$$

Proof. We begin to look for a weak solution to (4.11). In view of Poincaré inequality, we can choose as norm in $\hat{W}^{1,2}\left(\Omega_{0}\right)$ the one associated to the scalar product

$$
\left(\nabla \mathbf{v}_{1}, \nabla \mathbf{v}_{2}\right), \quad \mathbf{v}_{1}, \mathbf{v}_{2} \in \hat{\mathcal{W}}^{1,2}\left(\Omega_{0}\right) .
$$

Let us multiply both sides of $(4.11)_{1}$ by $\varphi \in \hat{\mathcal{W}}^{1,2}\left(\Omega_{0}\right)$ and integrate by parts over $\Omega_{0}$. Taking into account $(4.11)_{3,4}$ we show

$$
(\nabla \mathbf{v}, \nabla \boldsymbol{\varphi})=-(\boldsymbol{f}, \boldsymbol{\varphi})
$$

Now, by assumption, the right hand side of (4.14) defines a bounded linear functional on $\hat{\mathcal{W}}^{1,2}\left(\Omega_{0}\right)$, namely, an element of $\hat{\mathcal{W}}^{-1,2}\left(\Omega_{0}\right)$, and so, by Riesz theorem, there is one and only one $\mathbf{v} \in \hat{\mathcal{W}}^{1,2}\left(\Omega_{0}\right)$ satisfying (4.14). Moreover, by replacing $\boldsymbol{\varphi}$ with $\mathbf{v}$ in (4.14), we obtain

$$
\|\nabla \mathbf{v}\|_{2} \leq\|\boldsymbol{f}\|_{-1,2} \text {. }
$$

Following a classical procedure, we can now associate to the weak solution $\mathbf{v}$ a "pressure" field $Q \in L^{2}\left(\Omega_{0}\right)$ such that

$$
(\nabla \mathbf{v}, \nabla \boldsymbol{\psi})=-(\boldsymbol{f}, \boldsymbol{\psi})+\left(Q, \nabla \cdot\left(e^{-\beta z} \boldsymbol{\psi}\right)\right), \text { for all } \boldsymbol{\psi} \in W^{1,2}\left(\Omega_{0}\right) .
$$

Actually, following [9, Theorem III.5.3], to show (4.16) it is enough to show that for any $f \in L^{2}\left(\Omega_{0}\right)$ with $(f, 1)=0$, the problem

$$
\nabla \cdot\left(e^{-\beta z} \boldsymbol{\psi}\right)=f, \quad \boldsymbol{\psi} \in \hat{W}^{1,2}\left(\Omega_{0}\right),\|\nabla \boldsymbol{\psi}\|_{2} \leq C\|f\|_{2},
$$

has at least one solution. In order to solve (4.17), we take $\boldsymbol{\psi}=\nabla \psi$ where $\psi$ solves the following Neumann problem

$$
\Delta \psi-\beta \partial_{z} \psi=e^{\beta z} f \text { in } \Omega_{0}, \quad \partial_{z} \psi=0 \text { at } z=0,1 .
$$

The existence of $\psi$ with the required properties is then secured by Lemma 4.1 . We next notice that, by choosing in (4.17) $f=Q-(Q, 1)$, from (4.16), (4.15) and (4.17) it follows that

$$
\|Q\|_{2} \leq C\|\boldsymbol{f}\|_{-1,2} \text {. }
$$

Now, the difference quotient of $\boldsymbol{\varphi}$ in any of the horizontal directions $\mathbf{e}, \delta^{-\ell} \boldsymbol{\varphi}(x):=(\boldsymbol{\varphi}(x)-\boldsymbol{\varphi}(x-\ell \mathbf{e})) / \ell$, is also an element of $\hat{\mathcal{W}}^{1,2}\left(\Omega_{0}\right)$ and, as such, can be replaced in (4.14). Thus, by a standard argument and by (4.15) we show

$$
\left\|\nabla \delta^{\ell} \mathbf{v}\right\|_{2} \leq\left\|\delta^{\ell} \boldsymbol{f}\right\|_{-1,2} \leq C\|\boldsymbol{f}\|_{2},
$$

which, in turn, by the properties of the difference quotient, implies

$$
\nabla \nabla^{\prime} \mathbf{v} \in L^{2}\left(\Omega_{0}\right), \quad\left\|\nabla \nabla^{\prime} \mathbf{v}\right\|_{2} \leq C\|\boldsymbol{f}\|_{2},
$$


where $\nabla^{\prime}$ is the restriction of $\nabla$ to the $x^{\prime}$-variables. Using a similar argument on (4.16) and employing (4.19), (4.20) we show

$$
\nabla^{\prime} Q \in L^{2}\left(\Omega_{0}\right), \quad\left\|\nabla^{\prime} Q\right\|_{2} \leq C\|\boldsymbol{f}\|_{2} .
$$

From $(4.20)$ and $(4.11)_{2}$ it also follows

$$
\partial_{z}^{2} v^{z} \in L^{2}\left(\Omega_{0}\right), \quad\left\|\partial_{z}^{2} v^{z}\right\|_{2} \leq C\|\boldsymbol{f}\|_{2},
$$

which, once combined with (4.20) gives

$$
\Delta v^{z} \in L^{2}\left(\Omega_{0}\right), \quad\left\|\Delta v^{z}\right\|_{2} \leq C\|\boldsymbol{f}\|_{2} .
$$

We now choose in (4.16) $\boldsymbol{\psi}=\psi \mathbf{k}, \psi \in C_{0}^{\infty}\left(\Omega_{0}\right.$. Integrating by parts, and employing (4.22) it then follows

$$
\partial_{z} Q \in L^{2}\left(\Omega_{0}\right), \quad\left\|\partial_{z} Q\right\|_{2} \leq C\|\boldsymbol{f}\|_{2},
$$

which combined with (4.21) gives, in particular,

$$
\nabla Q \in L^{2}\left(\Omega_{0}\right), \quad\|\nabla Q\|_{2} \leq C\|\boldsymbol{f}\|_{2} .
$$

Inserting this information back into equation (4.16) with $\boldsymbol{\psi} \in C_{0}^{\infty}\left(\Omega_{0}\right)$ and integrating by parts, we finally conclude $\mathbf{v} \in W^{2,2}\left(\Omega_{0}\right)$, along with the validity of (4.12).

The next result is a corollary to Lemma 4.3.

Lemma 4.4. There exists an orthogonal basis $\left\{\boldsymbol{\Psi}_{j}\right\} \subset \hat{\mathcal{L}}^{2}\left(\Omega_{0}\right) \cap \hat{W}^{2,2}$ of $\hat{\mathcal{L}}^{2}\left(\Omega_{0}\right)$ constituted by eigenfuctions of the operator A, namely:

$$
\begin{aligned}
& \begin{array}{l}
\Delta \boldsymbol{\Psi}_{j}=-\lambda_{(j)} \boldsymbol{\Psi}_{j}+e^{-\beta z} \nabla Q_{j}, \quad \lambda_{(j)}>0 \\
\nabla \cdot\left(e^{-\beta z} \boldsymbol{\Psi}_{j}\right)=0 \\
\partial_{z} \Psi_{j}^{x^{\prime}}=0, \quad \Psi_{j}^{z}=0 \quad \text { at } z=0,1,
\end{array} \text { in } \Omega_{0},
\end{aligned}
$$

Proof. The previous lemma shows that the operator $A$ is surjective with a compact inverse. Since $A$ is symmetric, this implies that $A$ is selfadjoint with a purely discrete spectrum. The lemma is then a consequence of classical results.

We also have to generalize the Friedrichs inequality as follows.

Lemma 4.5. Let $\left\{\boldsymbol{\Psi}_{i}\right\}$ be a basis of $\hat{\mathcal{L}}^{2}\left(\Omega_{0}\right)$, and let $\mathbf{v} \in \hat{\mathcal{L}}^{2}\left(\Omega_{0}\right) \cap W^{1,2}\left(\Omega_{0}\right)$. Then, for any $\varepsilon>0$ there is $n=n(\varepsilon) \in \mathbb{N}$ such that

$$
\|\mathbf{v}\|_{2}^{2} \leq \varepsilon\|\nabla \mathbf{v}\|_{2}^{2}+C(n, \varepsilon) \sum_{i=1}^{n}\left|\left(e^{-\beta z} \mathbf{v}, \Psi_{i}\right)\right|^{2} .
$$

Proof. By [9, Lemma II.5.3], for any $\varepsilon>0$ there is $n=n(\varepsilon) \in \mathbb{N}$ such that

$$
\|\mathbf{v}\|_{2}^{2} \leq \varepsilon\|\nabla \mathbf{v}\|_{2}^{2}+C(n, \varepsilon) \sum_{i=1}^{n}\left|\ell_{i}(\mathbf{v})\right|^{2},
$$

where $\left\{\ell_{i}\right\}$ is a complete family of functionals on $\hat{\mathcal{L}}^{2}\left(\Omega_{0}\right) \cap W^{1,2}\left(\Omega_{0}\right)$, namely, $\ell_{i}(\mathbf{v})=0$ for all $i \in \mathbb{N}$ implies $\mathbf{v} \equiv 0$. If we choose

$$
\ell_{i}: \mathbf{v} \in \hat{\mathcal{L}}^{2}\left(\Omega_{0}\right) \cap W^{1,2}\left(\Omega_{0}\right) \mapsto \ell_{i}(\mathbf{v}):=\left(e^{-\beta z} \mathbf{v}, \Psi_{i}\right) \in \mathbb{R},
$$

by Lemma 4.2 it follows that $e^{-\beta z} \mathbf{v}=e^{-\beta z} \nabla Q$, for some $Q \in W^{1,2}\left(\Omega_{0}\right)$. However, by (2.11), this implies $\left(e^{-\beta z} \nabla Q, \nabla Q\right)=0$, namely, $\mathbf{v}=0$, which completes the proof of the lemma.

We end this section by recalling a number of classical inequalities that will be frequently employed and valid for a vector function $\mathbf{v}: \Omega_{0} \rightarrow \mathbb{R}^{n}, n=2,3$, satisfying (2.10). First of all, the Poincaré inequality

$$
\|\mathbf{v}\|_{2} \leq \gamma_{P}\|\nabla \mathbf{v}\|_{2},
$$


where $\gamma_{P}$ is a (positive) numerical constant. In what follows, we denote by the same symbol $C$ different constants depending, at most, on the domain $\Omega_{0}$. Integrating by parts over $\Omega_{0}$ one gets

$$
\|\nabla \mathbf{v}\|_{2}^{2}=-(\Delta \mathbf{v}, \mathbf{v})
$$

and so, by Poincaré and Schwarz inequalities, we deduce

$$
\|\nabla \mathbf{v}\|_{2} \leq C\|\Delta \mathbf{v}\|_{2}=C\left\|D^{2} \mathbf{v}\right\|_{2}
$$

since the norm of the Laplacian and that of all second derivatives coincide (see, e.g., [7, Lemma A.1]):

$$
\|\Delta \mathbf{v}\|_{2}=\left\|D^{2} \mathbf{v}\right\|_{2}
$$

Furthermore, in three dimensions, we have the Sobolev-Poincaré inequality

$$
\|\mathbf{v}\|_{6} \leq C\|\nabla \mathbf{v}\|_{2},
$$

and also

$$
\|\nabla \mathbf{v}\|_{6} \leq C\|\Delta \mathbf{v}\|_{2}
$$

whereas, by (4.25)-(4.27), in both two- and three-dimensional cases the Morrey inequality holds [7, Lemma A.3]:

$$
\|\mathbf{v}\|_{\infty} \leq C\|\Delta \mathbf{v}\|_{2}
$$

We recall Ladyzhenskaya's inequality (see [9]):

$$
\begin{aligned}
& r \in[2,4],\|\mathbf{v}\|_{r} \leq C\|\mathbf{v}\|_{2}^{1-a}\|\nabla \mathbf{v}\|_{2}^{a}, \quad n=2, a=\frac{r-2}{2 r}, \\
& r \in[2,6],\|\mathbf{v}\|_{r} \leq C\|\mathbf{v}\|_{2}^{1-b}\|\nabla \mathbf{v}\|_{2}^{b}, \quad n=3, b=\frac{3}{2} \frac{r-2}{2 r} .
\end{aligned}
$$

Combining the latter with Poincaré inequality, we infer, in particular,

$$
\|\mathbf{v}\|_{4} \leq C\|\nabla \mathbf{v}\|_{2}, \quad n=2,3 .
$$

Utilizing classical Sobolev's embedding in conjunction with (4.27), (4.26) and Poincaré inequality we can also show

$$
\begin{array}{ll}
r \in[2,4],\|\nabla \mathbf{v}\|_{r} \leq C\|\nabla \mathbf{v}\|_{2}^{1-a}\|\Delta \mathbf{v}\|_{2}^{a}, & n=2, a=\frac{2-r}{2 r} \\
r \in[2,6],\|\nabla \mathbf{v}\|_{r} \leq C\|\nabla \mathbf{v}\|_{2}^{1-b}\|\Delta \mathbf{v}\|_{2}^{b}, & n=3, \quad b=\frac{3}{2} \frac{r-2}{2 r}
\end{array}
$$

\section{Proof of Theorem 3.1}

We shall employ Galerkin method with the special basis $\left\{\boldsymbol{\Psi}_{j}\right\}$ introduced in Lemma 4.4. For each $N \in \mathbb{N}$, we look for an "approximate solution" defined by

$$
\mathbf{v}_{N}(x, t)=\sum_{j=1}^{N} C_{j}^{N}(t) \mathbf{\Psi}_{j}(\mathbf{x})
$$

where the coefficients $C_{j}^{N}(t)$ are searched as solutions to the the following system of ODE's:

$$
\sum_{j=1}^{N}\left(\frac{1}{\operatorname{Pr}} \dot{C}_{j}^{N}(t) B_{j k}+C_{j}^{N}(t) D_{j k}+\beta^{2} \gamma C_{j}^{N}(t)\left(\Psi_{j}^{z}, \Psi_{k}^{z}\right)\right)+\sum_{j, l=1}^{N} C_{j}^{N}(t) C_{l}^{N}(t) \Lambda_{j l k}=0,
$$

where

$$
B_{j k}:=\left(e^{-\beta z} \boldsymbol{\Psi}_{j}, \boldsymbol{\Psi}_{k}\right), \quad D_{j k}:=\left(\nabla \boldsymbol{\Psi}_{j}, \nabla \boldsymbol{\Psi}_{k}\right), \quad \Lambda_{j l k}:=\left(e^{-\beta z} \boldsymbol{\Psi}_{j} \cdot \nabla \boldsymbol{\Psi}_{l}, \mathbf{\Psi}_{k}\right),
$$

and $C_{j}^{N}(t)=C_{j}(0)$, for all $j, N \in \mathbb{N}$, where

$$
\mathbf{v}^{0}(\mathbf{x})=\sum_{j=1}^{\infty} C_{j}(0) \Psi_{j}(\mathbf{x})
$$


Since the matrix $B_{i j}$ is symmetric and, in view of (4.3), positive definite, the system of differential equations (5.1) can be put in normal form. Notice that (5.1) can be equivalently rewritten as

$$
\frac{1}{\operatorname{Pr}}\left(e^{-\beta z}\left(\mathbf{v}_{N}\right)_{t}, \Psi_{j}\right)+\frac{1}{\operatorname{Pr}}\left(e^{-\beta z} \mathbf{v}_{N} \cdot \nabla \mathbf{v}_{N}, \Psi_{j}\right)+\left(\nabla \mathbf{v}_{N}, \nabla \Psi_{j}\right)+\beta^{2} \gamma\left(v_{N}^{z}, \Psi_{j}^{z}\right)=0,
$$

Because the involved nonlinear terms are quadratic, it follows that for all $N \in \mathbb{N}$, (5.1) has one and only one solution $\mathbf{C}^{N}:=\left(C_{1}^{N}, \ldots, C_{N}^{N}\right)$ in some time interval $\left(0, T_{N}\right)$. Clearly, $T_{N}=\infty$ if we can show that $\left|\mathbf{C}^{N}(t)\right|$ is uniformly bounded. To this end, we multiply each side of the equation in (5.2) by $C_{j}^{N}$, sum over $j$ and argue as in (4.1) to get (since the basis function are regular)

$$
\frac{1}{2 \operatorname{Pr}} \frac{d}{d t}\left\|\mathbf{v}_{N}(t)\right\|_{2, \beta}^{2}+\left\|\nabla \mathbf{v}_{N}(t)\right\|_{2}^{2}+\gamma \beta^{2}\left\|v_{N}^{z}(t)\right\|_{2}^{2}=0 .
$$

Integrating both sides of (5.3) from 0 to $T_{N}$ and observing that $\left\|\mathbf{v}_{N}^{0}\right\|_{2, \beta} \leq C\left\|\mathbf{v}^{0}\right\|_{2}$, it follows at once the desired property for $\mathbf{C}^{N}$. Moreover, also with the help of (4.25), we also infer that the sequence $\left\{\mathbf{v}_{N}\right\}$ satisfies the following estimate

$$
\sup _{t \in(0, \infty)}\left\|\mathbf{v}_{N}(t)\right\|_{2}^{2}+C \int_{0}^{\infty}\left\|\mathbf{v}_{N}(t)\right\|_{1,2}^{2} d t \leq C(\operatorname{Pr})\left\|\mathbf{v}^{0}\right\|_{2}^{2} .
$$

In particular, the latter implies the existence of

$$
\mathbf{v} \in L^{\infty}\left(0, T ; \hat{\mathcal{L}}^{2}\left(\Omega_{0}\right)\right) \cap L^{2}\left(0, T ; W^{1,2}\left(\Omega_{0}\right)\right), \text { all } T>0,
$$

and of a subsequence $\left\{\mathbf{v}_{N_{k}}\right\}$ such that

$$
\mathbf{v}_{N_{k}} \rightarrow \mathbf{v} \text {, weakly in } L^{2}\left(0, T ; W^{1,2}\left(\Omega_{0}\right)\right) \text { and weak }{ }^{*} \text { in } L^{\infty}\left(0, T ; \hat{\mathcal{L}}^{2}\left(\Omega_{0}\right)\right) .
$$

It is easy to show that the latter, combined with (5.3), (4.25) and Gronwall's lemma, leads to (3.2). Next, by following more or less classical arguments in conjunction with Lemma 4.5, we shall show that

$$
\mathbf{v}_{N_{k}} \rightarrow \mathbf{v} \text { strongly in } L^{2}\left(0, T ; L^{2}\left(\Omega_{0}\right)\right) .
$$

Actually, from (5.2) and (4.30) we easily get with arbitrary $t_{1}$ and $t_{2}$, and $\boldsymbol{\Phi}_{j}:=e^{-\beta z} \boldsymbol{\Psi}_{j}$

$$
\begin{aligned}
& \frac{1}{\operatorname{Pr}}\left|\left(\mathbf{v}_{N_{k}}\left(t_{2}\right), \boldsymbol{\Phi}_{j}\right)-\left(\mathbf{v}_{N_{k}}\left(t_{1}\right), \mathbf{\Phi}_{j}\right)\right| \\
& \quad \leq\left|\int_{t_{1}}^{t_{2}}\left(e^{-\beta z} \mathbf{v}_{N_{k}} \cdot \nabla \mathbf{v}_{N_{k}}(t), \boldsymbol{\Psi}_{j}\right) d t\right|+\left|\int_{t_{1}}^{t_{2}}\left(\nabla \mathbf{v}_{N_{k}}(t), \nabla \boldsymbol{\Psi}_{j}\right) d t\right|+\beta^{2} \gamma\left|\int_{t_{1}}^{t_{2}}\left(v_{N_{k}}^{z}(t), \Psi_{j}^{z}\right) d t\right| \\
& \quad \leq\left\|\boldsymbol{\Psi}_{j}\right\|_{\infty} \int_{t_{1}}^{t_{2}}\left\|\mathbf{v}_{N_{k}}\right\|_{2}\left\|\nabla \mathbf{v}_{N_{k}}\right\|_{2} d t+\int_{t_{1}}^{t_{2}}\left\|\nabla \mathbf{v}_{N_{k}}(t)\right\|_{2}\left\|\nabla \Psi_{j}\right\|_{2} d t+\beta^{2} \gamma \int_{t_{1}}^{t_{2}}\left\|\mathbf{v}_{N_{k}}(t)\right\|_{2}\left\|\boldsymbol{\Psi}_{j}\right\|_{2} d t \\
& \quad \leq C\left\|\boldsymbol{\Psi}_{j}\right\|_{2,2}\|\mathbf{v}(0)\|_{2}\left(\left|t_{2}-t_{1}\right|+\left(\|\mathbf{v}(0)\|_{2}+1\right)\left|t_{2}-t_{1}\right|^{\frac{1}{2}}\right)
\end{aligned}
$$

From (5.8), by Cantor's diagonalization process one can show by standard methods (e.g. [8]) that

$$
\left(e^{-\beta z} \mathbf{v}_{N_{k}}(t), \Psi_{i}\right) \rightarrow\left(e^{-\beta z} \mathbf{v}(t), \Psi_{i}\right), \text { uniformly in } t, \text { forall } i \in \mathbb{N} .
$$

If we now apply (4.24) to $\mathbf{v}_{N_{k}}-\mathbf{v}$, integrate both sides of the resulting equation from 0 to $T$ and use (5.3), we get

$$
\int_{0}^{T}\left\|\mathbf{v}_{N_{k}}-\mathbf{v}\right\|_{2}^{2} d t \leq \varepsilon C(\operatorname{Pr})\left\|\mathbf{v}^{0}\right\|_{2}^{2}+C(\varepsilon, n) \sum_{i=1}^{n} \int_{0}^{T}\left|\left(e^{-\beta z}\left(\mathbf{v}_{N_{k}}-\mathbf{v}\right), \Psi_{i}\right)\right|^{2} d t .
$$

Thus, letting $N_{k} \rightarrow \infty$ in this relation and employing (5.9), by the arbitrariness of $\varepsilon$ we arrive at (5.7). From now on the procedure to prove that $\mathbf{v}$ is in fact the weak solution is fully standard: we first observe 
that from (5.2) it follows that

$$
\begin{aligned}
\frac{1}{\operatorname{Pr}}\left(\mathbf{v}_{N_{k}}(t), \boldsymbol{\Phi}_{j}\right)+\int_{0}^{t}\left(e^{-\beta z} \mathbf{v}_{N_{k}} \cdot \nabla \mathbf{v}_{N_{k}}(s), \mathbf{\Psi}_{j}\right) d s & +\int_{0}^{t}\left(\nabla \mathbf{v}_{N_{k}}(s), \nabla \mathbf{\Psi}_{j}\right) d s \\
& +\beta^{2} \gamma \int_{0}^{t}\left(v_{N_{k}}^{z}(s), \Psi_{j}^{z}\right) d s=\frac{1}{\operatorname{Pr}}\left(\mathbf{v}_{N_{k}}^{0}, \boldsymbol{\Phi}_{j}\right) .
\end{aligned}
$$

If we let $N_{k} \rightarrow \infty$ in (5.10) we can show, in view of (5.6), that the limit function $\mathbf{v}$ satisfies (3.1) with $\boldsymbol{\Psi} \equiv \boldsymbol{\Psi}_{j}$, provided we also prove

$$
\int_{0}^{t}\left(\mathbf{v}_{N_{k}} \cdot \nabla \mathbf{v}_{N_{k}}, \Phi_{j}\right) \rightarrow \int_{0}^{t}\left(\mathbf{v} \cdot \nabla \mathbf{v}, \Phi_{j}\right)
$$

or, equivalently,

$$
\lim _{N_{k} \rightarrow \infty}\left(\int_{0}^{t}\left(\left(\mathbf{v}_{N_{k}}-\mathbf{v}\right) \cdot \nabla \mathbf{v}_{N_{k}}(s), \boldsymbol{\Phi}_{j}\right) d s+\int_{0}^{t}\left(\mathbf{v} \cdot \nabla\left(\mathbf{v}_{N_{k}}-\mathbf{v}\right)(s), \Phi_{j}\right) d s\right)=0 .
$$

In view of (5.6), it follows that the second term on the left hand side goes to zero. Concerning the fisrst one, we notice that by Hölder and Sobolev inequalities, we deduce

$$
\left|\int_{0}^{t}\left(\left(\mathbf{v}_{N_{k}}-\mathbf{v}\right) \cdot \nabla \mathbf{v}_{N_{k}}, \Phi_{j}\right)\right| \leq \int_{0}^{t}\left\|\mathbf{v}_{N_{k}}-\mathbf{v}\right\|_{4}\left\|\nabla \mathbf{v}_{N_{k}}\right\|\left\|_{2}\right\| \Phi_{j}\left\|_{4} \leq\right\| \Phi_{j}\left\|_{1,2} \int_{0}^{t}\right\| \mathbf{v}_{N_{k}}-\mathbf{v}\left\|_{4}\right\| \nabla \mathbf{v}_{N_{k}} \|_{2} .
$$

Next, from $(4.31)_{1}$ and $(4.31)_{2}$ we infer that the right hand side in this inequality can be increased by

$$
\begin{aligned}
& C\left\|\boldsymbol{\Phi}_{j}\right\|_{1,2} \int_{0}^{t}\left\|\mathbf{v}_{N_{k}}-\mathbf{v}\right\|_{2}^{\frac{1}{2}}\left\|\nabla \mathbf{v}_{N_{k}}-\nabla \mathbf{v}\right\|_{2}^{\frac{1}{2}}\left\|\nabla \mathbf{v}_{N_{k}}\right\|_{2}, \quad n=2, \\
& C\left\|\boldsymbol{\Phi}_{j}\right\|_{1,2} \int_{0}^{t}\left\|\mathbf{v}_{N_{k}}-\mathbf{v}\right\|_{2}^{\frac{1}{4}}\left\|\nabla \mathbf{v}_{N_{k}}-\nabla \mathbf{v}\right\|_{2}^{\frac{3}{4}}\left\|\nabla \mathbf{v}_{N_{k}}\right\|_{2}, \quad n=3 .
\end{aligned}
$$

Thus, employing with Hölder inequality with exponents $(4,4,2)$ for $n=2$ and $\left(8, \frac{8}{3}, 2\right)$ for $n=3$, with the help of (5.4) we obtain

$$
\begin{array}{r}
\left|\int_{0}^{t}\left(\left(\mathbf{v}_{N_{k}}-\mathbf{v}\right) \cdot \nabla \mathbf{v}_{N_{k}}, \boldsymbol{\Phi}_{j}\right)\right| \leq C\left\|\boldsymbol{\Phi}_{j}\right\|_{1,2}\left(\int_{0}^{t}\left\|\mathbf{v}_{N_{k}}-\mathbf{v}\right\|_{2}^{2}\right)^{\frac{1}{4}}\left(\int_{0}^{t}\left\|\nabla \mathbf{v}_{N_{k}}-\nabla \mathbf{v}\right\|_{2}^{2}\right)^{\frac{1}{4}}\left(\int_{0}^{t}\left\|\nabla \mathbf{v}_{N_{k}}\right\|_{2}^{2}\right)^{\frac{1}{2}} \\
\leq C\|\boldsymbol{\Phi}\|_{1,2}\left(\int_{0}^{t}\left\|\mathbf{v}_{N_{k}}-\mathbf{v}\right\|_{2}^{2}\right)^{\frac{1}{4}}\|\mathbf{v}(0)\|_{2}^{\frac{5}{4}} n=2, \\
\left|\int_{0}^{t}\left(\left(\mathbf{v}_{N_{k}}-\mathbf{v}\right) \cdot \nabla \mathbf{v}_{N_{k}}, \mathbf{\Phi}_{j}\right)\right| \leq C\left\|\mathbf{\Phi}_{j}\right\|_{1,2}\left(\int_{0}^{t}\left\|\mathbf{v}_{N_{k}}-\mathbf{v}\right\|_{2}^{2}\right)^{\frac{1}{8}}\left(\int_{0}^{t}\left\|\nabla \mathbf{v}_{N_{k}}-\nabla \mathbf{v}\right\|_{2}^{2}\right)^{\frac{3}{8}}\left(\int_{0}^{t}\left\|\nabla \mathbf{v}_{N_{k}}\right\|_{2}^{2}\right)^{\frac{1}{2}} \\
\leq C\left\|\boldsymbol{\Phi}_{j}\right\|_{1,2}\left(\int_{0}^{t}\left\|\mathbf{v}_{N_{k}}-\mathbf{v}\right\|_{2}^{2}\right)^{\frac{1}{8}}\|\mathbf{v}(0)\|_{2}^{\frac{7}{4}} n=3 .
\end{array}
$$

The last bounds, combined with (5.7), imply (5.11). We may thus conclude that the field $\mathbf{v}$ satisfies (3.1) with $\boldsymbol{\Psi} \equiv \boldsymbol{\Psi}_{j}$, for all $j \in \mathbb{N}$. Since $\left\{\boldsymbol{\Psi}_{j}\right\}$ is complete in $\hat{\mathcal{W}}^{1,2}\left(\Omega_{0}\right)$ then, by a standard argument, one shows that (3.1) is, in fact, satisfied for all $\boldsymbol{\Psi} \in \hat{\mathcal{W}}^{1,2}\left(\Omega_{0}\right)$, which completes the proof.

\section{Proofs of Theorems 3.2 and 3.3}

In this section we shall show that, provided the initial data are more regular, the corresponding Galerkin approximation $\mathbf{v}_{N}$ belongs, uniformly in $N$, to a better regularity class (the so-called "Prodi class"). This will be achieved through suitable "energy estimates" that will eventually lead to the proofs of Theorems 
3.2 and 3.3. In this regard, we need some preliminary results. By multiplying both sides of (5.2) by $\dot{C}_{j}^{N}$, sum over $j$ from 1 to $N$ and integrate by parts as necessary to infer

$$
\frac{1}{2} \frac{d}{d t}\left(\left\|\nabla \mathbf{v}_{N}(t)\right\|_{2}^{2}+\gamma \beta^{2}\left\|v_{N}^{z}(t)\right\|_{2}^{2}\right)+\frac{1}{\operatorname{Pr}}\left\|\left(\mathbf{v}_{N}\right)_{t}(t)\right\|_{2, \beta}^{2}=-\frac{1}{\operatorname{Pr}}\left(e^{-\beta z} \mathbf{v}_{N} \cdot \nabla \mathbf{v}_{N},\left(\mathbf{v}_{N}\right)_{t}\right) .
$$

Likewise, multiplying both sides of $(5.2)$ by $-\lambda_{(j)} C_{j}^{N}$, summing over $j$ from 1 to $N$ the resulting equation is

$$
\frac{1}{\operatorname{Pr}}\left(e^{-\beta z}\left(\mathbf{v}_{N}\right)_{t}, P \Delta \mathbf{v}_{N}\right)-\left\|P \Delta \mathbf{v}_{N}\right\|_{2}^{2}-\beta^{2} \gamma\left(\mathbf{v}_{N}, \mathbf{k} \cdot P \Delta v_{N}\right)=-\frac{1}{\operatorname{Pr}}\left(e^{-\beta z} \mathbf{v}_{N} \cdot \nabla \mathbf{v}_{N}, P \Delta \mathbf{v}_{N}\right) .
$$

Our next task is to give a suitable estimate of the terms on the right hand side of (6.1) and (6.2). To this end, we observe that, by Lemma 4.3, we have

$$
\left\|\Delta \mathbf{v}_{N}\right\|_{2} \geq\left\|P \Delta \mathbf{v}_{N}\right\|_{2} \geq C_{0}\left\|\Delta \mathbf{v}_{N}\right\|_{2} .
$$

with $C$ independent of $N$.

Lemma 6.1. Let $\mathbf{v}_{N}$ be a Galerkin solution (3.1), for arbitrary $\epsilon_{i}>0, i=1,2$, if $n=2$, then

$$
\left|\left(e^{-\beta z} \mathbf{v}_{N} \cdot \nabla \mathbf{v}_{N},\left(\mathbf{v}_{N}\right)_{t}\right)\right| \leq C_{\epsilon_{1}, \epsilon_{2}}\left\|\mathbf{v}_{N}\right\|_{2}^{2}\left\|\nabla \mathbf{v}_{N}\right\|_{2}^{4}+\epsilon_{2}\left\|\Delta \mathbf{v}_{N}\right\|_{2}^{2}+\epsilon_{1}\left\|\left(\mathbf{v}_{N}\right)_{t}\right\|_{2, \beta}^{2},
$$

while if $n=3$, then

$$
\left|\left(e^{-\beta z} \mathbf{v}_{N} \cdot \nabla \mathbf{v}_{N},\left(\mathbf{v}_{N}\right)_{t}\right)\right| \leq C_{\epsilon_{1}, \epsilon_{2}}\left\|\nabla \mathbf{v}_{N}\right\|_{2}^{3}+\epsilon_{2}\left\|\Delta \mathbf{v}_{N}\right\|_{2}+\epsilon_{1}\left\|\left(\mathbf{v}_{N}\right)_{t}\right\|_{2}^{2} .
$$

Proof. By using Hölder inequality, $(4.31)_{1},(4.33)_{1}$ and Young inequality, for $n=2$ one deduces for arbitrary $\epsilon_{1}, \epsilon_{2}>0$

$$
\begin{aligned}
& \left|\left(e^{-\beta z} \mathbf{v}_{N} \cdot \nabla \mathbf{v}_{N},\left(\mathbf{v}_{N}\right)_{t}\right)\right| \leq C\left\|\mathbf{v}_{N}\right\|_{4}\left\|\nabla \mathbf{v}_{N}\right\|_{4}\left\|\left(\mathbf{v}_{N}\right)_{t}\right\|_{2} \\
& \quad \leq C_{\epsilon_{1}}\left\|\mathbf{v}_{N}\right\|_{4}^{2}\left\|\nabla \mathbf{v}_{N}\right\|_{4}^{2}+\epsilon_{1}\left\|\left(\mathbf{v}_{N}\right)_{t}\right\|_{2}^{2} \leq C_{\epsilon_{1}}\left\|\mathbf{v}_{N}\right\|_{2}\left\|\nabla \mathbf{v}_{N}\right\|_{2}^{2}\left\|\Delta \mathbf{v}_{N}\right\|_{2}+\epsilon_{1}\left\|\left(\mathbf{v}_{N}\right)_{t}\right\|_{2}^{2} \\
& \quad \leq C_{\epsilon_{1}, \epsilon_{2}}\left\|\mathbf{v}_{N}\right\|_{2}^{2}\left\|\nabla \mathbf{v}_{N}\right\|_{2}^{4}+\epsilon_{2}\left\|\Delta \mathbf{v}_{N}\right\|_{2}^{2}+\epsilon_{1}\left\|\left(\mathbf{v}_{N}\right)_{t}\right\|_{2, \beta}^{2} .
\end{aligned}
$$

For $n=3$, by (4.28), (4.33) 2 , and Young inequalities, we show

$$
\begin{aligned}
\left|\left(e^{-\beta z} \mathbf{v}_{N} \cdot \nabla \mathbf{v}_{N},\left(\mathbf{v}_{N}\right)_{t}\right)\right| & \leq\left\|\mathbf{v}_{N}\right\|\left\|_{6}\right\| \nabla \mathbf{v}_{N}\left\|_{3}\right\|\left(\mathbf{v}_{N}\right)_{t} \|_{2} \\
& \leq C\left\|\nabla \mathbf{v}_{N}\right\|_{2}^{\frac{3}{2}}\left\|\Delta \mathbf{v}_{N}\right\|_{2}^{\frac{1}{2}}\left\|\left(\mathbf{v}_{N}\right)_{t}\right\|_{2} \\
& \leq C_{\epsilon_{1}, \epsilon_{2}}\left\|\nabla \mathbf{v}_{N}\right\|_{2}^{6}+\epsilon_{2}\left\|\Delta \mathbf{v}_{N}\right\|_{2}^{2}+\epsilon_{1}\left\|\left(\mathbf{v}_{N}\right)_{t}\right\|_{2}^{2}
\end{aligned}
$$

Lemma 6.2. Uniformly in $N$ and in $t>0$ we get

$$
\begin{aligned}
& \frac{d}{d t}\left\|\nabla v_{N}(t)\right\|_{2}^{2}+c_{1}\left\|\left(v_{N}\right)_{t}\right\|_{2}^{2}+c_{2}\left\|P \Delta v_{N}\right\|_{2}^{2} \\
& c\left|\left(e^{-\beta z} \mathbf{v}_{N} \cdot \nabla \mathbf{v}_{N},\left(\mathbf{v}_{N}\right)_{t}\right)\right|+c\left|\left(e^{-\beta z} \mathbf{v}_{N} \cdot \nabla \mathbf{v}_{N}, P \Delta \mathbf{v}_{N}\right)\right| .
\end{aligned}
$$

Proof. From formula (6.2), via Hölder's and Young inequality we easily get

$$
\left\|P \Delta \mathbf{v}_{N}\right\|_{2}^{2} \leq 2\left\|\left(\mathbf{v}_{N}\right)_{t}\right\|^{2}+2\left(\beta^{2} \gamma\right)^{2}\left\|\mathbf{v}_{N}, \mathbf{k}\right\|^{2}+c_{0}\left|\frac{1}{\operatorname{Pr}}\left(e^{-\beta z} \mathbf{v}_{N} \cdot \nabla \mathbf{v}_{N}, P \Delta \mathbf{v}_{N}\right)\right| .
$$

Multiplying (6.7) by a suitable constant and summing to (6.1), we arrive at (6.6).

Lemma 6.3. Let $\mathbf{v}_{N}$ be a Galerkin solution (3.1), for arbitrary $\epsilon_{3}>0$, if $n=2$, then

$$
\left|\left(e^{-\beta z} \mathbf{v}_{N} \cdot \nabla \mathbf{v}_{N}, P \Delta \mathbf{v}_{N}\right)\right| \leq C_{\epsilon_{3}}\left\|\mathbf{v}_{N}\right\|_{2}^{2}\left\|\nabla \mathbf{v}_{N}\right\|_{2}^{4}+\epsilon_{3}\left\|\Delta \mathbf{v}_{N}\right\|_{2}^{2},
$$

while if $n=3$, then

$$
\left|\left(\mathbf{v}_{N} \cdot \nabla \mathbf{v}_{N}, e^{-\beta z} \Delta \mathbf{v}_{N}\right)\right| \leq C_{\epsilon_{3}}\left\|\nabla \mathbf{v}_{N}\right\|_{2}^{6}+\epsilon_{3}\left\|\Delta \mathbf{v}_{N}\right\|_{2}^{2}
$$


Proof. As is done above, and with the help of (6.3), for any $\epsilon_{3}>0$ we show

$$
\begin{aligned}
& \left|\left(e^{-\beta z} \mathbf{v}_{N} \cdot \nabla \mathbf{v}_{N}, P \Delta \mathbf{v}_{N}\right)\right| \leq C\left\|\mathbf{v}_{N}\right\|\left\|_{4}\right\| \nabla \mathbf{v}_{N}\left\|_{4}\right\| \Delta \mathbf{v}_{N} \|_{2} \\
& \quad \leq C\left\|\mathbf{v}_{N}\right\|_{2}^{1 / 2}\left\|\nabla \mathbf{v}_{N}\right\|_{2}\left\|\Delta \mathbf{v}_{N}\right\|_{2}^{3 / 2} \leq C_{\epsilon_{3}}\left\|\mathbf{v}_{N}\right\|_{2}^{2}\left\|\nabla \mathbf{v}_{N}\right\|_{2}^{4}+\epsilon_{3}\left\|\Delta \mathbf{v}_{N}\right\|_{2}^{2} .
\end{aligned}
$$

For $n=3$, by (4.28), (4.33) $)_{2}$, and Young inequalities, we show

$$
\begin{aligned}
\left|\left(\mathbf{v}_{N} \cdot \nabla \mathbf{v}_{N}, e^{-\beta z} \Delta \mathbf{v}_{N}\right)\right| & \leq\left\|\mathbf{v}_{N}\right\|_{6}\left\|\nabla \mathbf{v}_{N}\right\|_{3}\left\|\Delta \mathbf{v}_{N}\right\|_{2} \\
& \leq C_{\epsilon_{3}}\left\|\mathbf{v}_{N}\right\|_{2}^{6}\left\|\nabla \mathbf{v}_{N}\right\|_{2}^{6}+\epsilon_{3}\left\|\Delta \mathbf{v}_{N}\right\|_{2}^{2} .
\end{aligned}
$$

Proof of Theorem 3.2. For $\lambda_{1}, \lambda_{2}>0$, let us set

$$
E:=\frac{\lambda_{1}}{2 \operatorname{Pr}}\left\|\mathbf{v}_{N}\right\|_{2}^{2}+\frac{\lambda_{2}}{2 \operatorname{Pr}}\left\|\nabla \mathbf{v}_{N}\right\|_{2}^{2}+\frac{\beta^{2} \gamma}{2}\left\|v_{N}^{z}\right\|_{2}^{2},
$$

and perform $\lambda(5.3)+(6.1)-(6.2)$. Taking into account (6.3)- (6.4), and choosing $\epsilon_{2}+2 \epsilon_{3}=(1 / 2) C_{0}$, we show

$$
\begin{aligned}
& \frac{d E}{d t} \leq-\left(\lambda_{1}-C_{\epsilon_{3}}\right)\left\|\nabla \mathbf{v}_{N}\right\|_{2}^{2}-\left(\frac{\lambda_{2}}{2 \operatorname{Pr}}-\epsilon_{1}-C_{\epsilon_{3}}\right)\left\|\left(\mathbf{v}_{N}\right)_{t}\right\|_{2}^{2} \\
& -\frac{C_{0}}{2}\left\|\Delta \mathbf{v}_{N}\right\|_{2}^{2}-\beta^{2} \gamma\left\|\nabla v_{N}^{z}\right\|_{2}^{2}+C\left(1+\left\|\mathbf{v}_{N}\right\|_{2}^{2}\right)\left\|\nabla \mathbf{v}_{N}\right\|_{2}^{4} .
\end{aligned}
$$

If we take $\lambda_{1}>C_{\epsilon_{3}}$ and $\lambda_{2}>2 \operatorname{Pr}\left(\epsilon_{1}+C_{\epsilon_{3}}\right)$, from (6.10) by Gronwall's lemma we infer, in particular,

$$
E(t) \leq E(0) \exp \left(\int_{0}^{t}\left(1+\left\|\mathbf{v}_{N}(s)\right\|_{2}^{2}\right)\left\|\nabla \mathbf{v}_{N}(s)\right\|_{2}^{2} d s\right) .
$$

We now observe that $\left\|\nabla \mathbf{v}_{N}^{0}\right\|_{2} \leq\left\|\nabla \mathbf{v}^{0}\right\|_{2}$ because $\left\{\boldsymbol{\Psi}_{j}\right\}$ is orthogonal in $\hat{\mathcal{L}}^{2}\left(\Omega_{0}\right)$ and, by (4.23)

$$
\mathbf{v}_{N}^{0}=\sum_{j=1}^{N}\left(\mathbf{v}_{0}, \boldsymbol{\Psi}_{j}\right) \boldsymbol{\Psi}_{j}=\sum_{j=1}^{N} \frac{1}{\left\|\nabla \boldsymbol{\Psi}_{j}\right\|_{2}^{2}}\left(\nabla \mathbf{v}_{0}, \nabla \boldsymbol{\Psi}_{j}\right) \boldsymbol{\Psi}_{j} .
$$

As a result, $E(0) \leq C\left\|\mathbf{v}^{0}\right\|_{1,2}^{2}$ and so, if the initial data are chosen in $\hat{\mathcal{W}}^{1,2}\left(\Omega_{0}\right)$, thanks to (5.4), from the previous relation we deduce

$$
\sup _{t \geq 0}\left\|\mathbf{v}_{N}(t)\right\|_{1,2} \leq C_{0}
$$

where $C_{0}$ depends only on the physical parameters and the norm of the initial data. If we replace this information back into (6.10) and integrate over $t \in(0, \infty)$ we also infer

$$
\int_{0}^{\infty}\left(\left\|\left(\mathbf{v}_{N}\right)_{t}(t)\right\|_{2}^{2}+\left\|\Delta \mathbf{v}_{N}(t)\right\|_{2}^{2}\right) d t \leq C_{0} .
$$

we thus conclude that the limiting field $\mathbf{v}$ defined in (5.5) satisfies the further property

$$
\mathbf{v} \in C\left([0, T) ; W^{1,2}\left(\Omega_{0}\right)\right) \cap L^{2}\left(0, T ; W^{2,2}\left(\Omega_{0}\right)\right), \quad \mathbf{v}_{t} \in L^{2}\left(0, T ; \mathcal{L}^{2}\left(\Omega_{0}\right)\right) .
$$

Now, for the existence part, we only have to prove the statement about the "pressure" П. Taking the time derivative of both sides of (3.1), we show for a.a. $t \in(0, T), T>0$,

$$
\left(\frac{1}{\operatorname{Pr}}\left(e^{-\beta z} \mathbf{v}_{t}+e^{-\beta z} \mathbf{v} \cdot \nabla \mathbf{v}\right)-\Delta \mathbf{v}+\gamma \beta^{2} v^{z} \mathbf{k}, \Psi\right)=0,
$$

for all $\boldsymbol{\Psi} \in \hat{\mathcal{W}}^{1,2}\left(\Omega_{0}\right)$. From this relation and Lemma 4.2, it immediately follows the existence of a function $\Pi$ with the stated properties. 
Now, we show uniqueness: let $(\mathbf{v}, \Pi)$ and $(\mathbf{v}+\mathbf{w}, \Pi+Q)$ be two solutions in the stated function class corresponding to the same initial data. Then, the "difference solution" $(\mathbf{w}, Q)$ satisfies the following equations

$$
\left\{\begin{array}{l}
\nabla \cdot\left(e^{-\beta z} \mathbf{w}\right)=0 \\
\frac{e^{-\beta z}}{\operatorname{Pr}}\left(\mathbf{w}_{t}+\mathbf{w} \cdot \nabla \mathbf{w}+\mathbf{v} \cdot \nabla \mathbf{w}+\mathbf{w} \cdot \nabla \mathbf{v}\right)-\Delta \mathbf{w}=-e^{\beta z} \nabla Q-\gamma \beta^{2} u^{z} \mathbf{k},
\end{array}\right.
$$

Thus, dot-multiplying both sides by $\mathbf{w}$, integrating by parts over $\Omega_{0}$ and using $(6.14)_{1}$, and then integrating the resulting equation over $(0, t), t<T$, we show

$$
\frac{1}{\operatorname{Pr}}\|\mathbf{w}(t)\|_{2, \beta}^{2}+\int_{0}^{t}\left(\|\nabla \mathbf{w}(s)\|_{2}^{2}+\gamma \beta^{2}\left\|w^{z}(s)\right\|_{2}^{2}\right) d s=-\int_{0}^{t}\left(e^{-\beta z} \mathbf{w}(s) \cdot \nabla \mathbf{v}(s), \mathbf{w}(s)\right) d s .
$$

From $(4.31)_{1}$ and the property $\mathbf{v} \in C\left([0, T] ; W^{1,2}\left(\Omega_{0}\right)\right.$, it follows that

$$
\begin{aligned}
-\int_{0}^{t}\left(e^{-\beta z} \mathbf{w}(s) \cdot \nabla \mathbf{v}(s), \mathbf{w}(s)\right) d s & \leq \max _{t \in[0, T]}\|\nabla \mathbf{v}(t)\|_{2} \int_{0}^{t}\|\mathbf{w}(s)\|_{4}^{2} d s \\
& \leq C \max _{t \in[0, T]}\|\nabla \mathbf{v}(t)\|_{2} \int_{0}^{t}\|\mathbf{w}(s)\|_{2}\|\nabla \mathbf{w}(s)\|_{2} d s .
\end{aligned}
$$

Therefore, applying Cauchy-Schwarz inequality on the last term of the latter and replacing the outcome in (6.15) we get, in particular,

$$
\|\mathbf{w}(t)\|_{2}^{2} \leq C \max _{t \in[0, T]}\|\nabla \mathbf{v}(t)\|_{2}^{2} \int_{0}^{t}\|\mathbf{w}(s)\|_{2}^{2} d s, \quad t \in[0, T],
$$

which, in turn, by Gronwall's lemma, implies w $\equiv 0$.

Proof of Theorem 3.3. We proceed in a slightly different way with respect to the previous two-dimensional case. We consider (6.6) and increase the right hand side by means of (6.4) and (6.9), choosing suitably $\epsilon_{i}, i=1,2,3$, we deduce the following differential inequality in $D_{N}(t):=\left\|\nabla v_{N}(t)\right\|_{2}^{2}$ :

$$
\frac{d D_{N}}{d t}+\kappa_{1}\left\|\Delta \mathbf{v}_{N}(t)\right\|_{2}^{2}+\kappa_{2}\left\|\left(\mathbf{v}_{N}\right)_{t}(t)\right\|_{2}^{2} \leq C_{1} D_{N}^{3} \text {, for all } N \text { and } t>0 .
$$

where the quantities $\kappa_{i}, i=1,2$, and $C_{1}$ depend only on the physical parameters and $\epsilon_{i}, i=1,2,3$. We integrate the differential inequality (6.16) two times. The former concerns simply a bound for $D(t)$. This bound is on some $(0, T)$ for arbitrary data. Instead the bound is global, that is $(0, \infty)$ for initial data small in the sense indicated in the statement. Without considering the "dissipative terms", by integrating we obtain

$$
-\frac{1}{D_{N}^{2}(t)}+\frac{1}{D_{N}^{2}(0)} \leq 2 C_{0} t \Leftrightarrow D_{N}(t) \leq D_{N}(0)\left[1-2 C_{0}\|\nabla v(0)\|_{2}^{4} t\right]^{-\frac{1}{2}}, t \in[0, T), \text { for all } N,
$$

where we set $T:=1 / 2 C_{0}\|\nabla v(0)\|_{2}^{2}$. In the case of small data we modify the integration as follows:

$$
\begin{aligned}
& -\frac{1}{D_{N}(t)}+\frac{1}{D_{N}(0)} \leq 2 C_{1} \int_{0}^{t} D_{N}(s) d s \\
& \quad \Rightarrow D_{N}(t) \leq D_{N}(0)\left[1-2 C_{0}\|\mathbf{v}(0)\|_{2}^{2}\|\nabla v(0)\|_{2}^{2}\right]^{-1}, t \in[0, \infty) \text {, for all } N,
\end{aligned}
$$

where we have taken into account (5.4) of the energy inequality. As a result, we have two uniform bounds (one local and the other global in time) for the sequence $\left\{\left\|\nabla v_{N}(t)\right\|\right\}$. Hence, integrating both sides of (6.16) we get, in particular,

$$
D_{N}(t)+\kappa_{1} \int_{0}^{t}\left\|\Delta v_{N}(s)\right\|_{2}^{2} d s+\kappa_{2} \int_{0}^{t}\left\|\left(v_{N}(s)\right)_{s}\right\|_{2}^{2} d s \leq C_{1} \int_{0}^{T} D_{N}^{3}(s) d s, t \in[0, T), \text { for all } N,
$$

where $T$ is finite or infinite depending on the "size" data. Proceeding exactly as in the case $n=2$, one can show that the approximating solutions $\left\{\mathbf{v}_{N}\right\}$ satisfies (6.11) and (6.12), thus implying that the limit field $\mathbf{v}$ is in the class $(6.13)$. 
The existence of a "pressure field" $\Pi$ satisfying the stated properties is proved exactly as in the proof of Theorem 3.2. As for uniqueness, also in this case we derive (6.15). Concerning the estimate of the nonlinear term, this time we use $(4.31)_{2}$ in conjunction with Young inequality to get

$$
\begin{aligned}
-\int_{0}^{t}\left(e^{-\beta z} \mathbf{w}(s) \cdot \nabla \mathbf{v}(s), \mathbf{w}(s)\right) d s & \leq C \max _{t \in[0, T]}\|\nabla \mathbf{v}(t)\|_{2} \int_{0}^{t}\|\mathbf{w}(s)\|_{2}^{\frac{1}{2}}\|\nabla \mathbf{w}(s)\|_{2}^{\frac{3}{2}} d s \\
& \leq \frac{1}{2} \int_{0}^{t}\|\nabla \mathbf{w}(s)\|_{2}^{2} d s+C \max _{t \in[0, T]}\|\nabla \mathbf{v}(t)\|_{2}^{4} \int_{0}^{t}\|\mathbf{w}(s)\|_{2}^{2} d s .
\end{aligned}
$$

As a result, replacing the latter into (6.15) we may argue exactly as in Theorem 3.2 to show $\mathbf{w} \equiv 0$.

Funding Open access funding provided by Universitá degli Studi di Ferrara within the CRUI-CARE Agreement.

\section{Declarations}

Conflict of interest The author declares that she has no conflict of interest.

Open Access. This article is licensed under a Creative Commons Attribution 4.0 International License, which permits use, sharing, adaptation, distribution and reproduction in any medium or format, as long as you give appropriate credit to the original author(s) and the source, provide a link to the Creative Commons licence, and indicate if changes were made. The images or other third party material in this article are included in the article's Creative Commons licence, unless indicated otherwise in a credit line to the material. If material is not included in the article's Creative Commons licence and your intended use is not permitted by statutory regulation or exceeds the permitted use, you will need to obtain permission directly from the copyright holder. To view a copy of this licence, visit http://creativecommons.org/licenses/by/4.0/.

Publisher's Note Springer Nature remains neutral with regard to jurisdictional claims in published maps and institutional affiliations.

\section{References}

[1] Chandrasekhar, S.: Hydrodynamic and Hydromagnetic Stability, On the Oberbeck-Boussinesq Approximation. Oxford University Press/Clarendon Press, Oxford (1961)

[2] Corli, A., Passerini, A.: The Bénard problem for slightly compressible materials: existence and linear instability. Mediterr. J. Math. 16, 17-40 (2019)

[3] Crispo, F., Grisanti, C.R., Maremonti, P.: Some properties of a suitable weak solution to the Navier-Stokes equations. Lecture Notes in Mathematical Fluid Mechanics, Waves in Flows, The 2018 Prague-Sum Workshop Lectures, pp. 159$179(2021)$

[4] De Martino, A., Passerini, A.: Existence and non linear stability of convective solutions for almost compressible fluids in Bénard problem. J. Math. Phys. (2019). https://doi.org/10.1063/1.5102063

[5] De Martino, A., Passerini, A.: A Lorenz model for almost compressible fluids. Mediterr. J. Math. 17(7), 1-12 (2020)

[6] Feireisl, E., Malek, J., Novotny, A.: An elastic approximation as a singular limit of the compressible Navier-Stokes system. Commun. Part. Differ. Equ. 33(1), 157-176 (2008)

[7] Galdi, G.P.: Nonlinear stability of the magnetic Bénard problem via a generalized energy method. Arch. Ration. Mech. Anal. 87, 167-186 (1985)

[8] Galdi, G.P.: An introduction to the Navier-Stokes initial-boundary value problem. In: Fundamental Directions in Mathematical Fluid Mechanics, pp. 1-70. Adv. Math. Fluid Mech., Birkhäuser, Basel (2000)

[9] Galdi, G.P.: An introduction to the mathematical Theory of the Navier-Stokes equation: Steady-State Problems. Springer, New York (2011)

[10] Gouin, H., Muracchini, A., Ruggeri, T.: On the Müller paradox for thermal-incompressible media. Contin. Mech. Therm. 24, 505-513 (2012)

[11] Gouin, H., Ruggeri, T.: A consistent thermodynamical model of incompressible media as limit case of quasi-thermalincompressible materials. Int. J. Nonlinear Mech. 47, 688-693 (2012)

[12] Grandi, G., Passerini, A.: On the Oberbeck-Boussinesq approximation for gases. Int. J. Nonlinear Mech. 134, (2021)

[13] Grandi, G., Passerini, A.: Approximation à la Oberbeck-Boussinesq for fluids with pressure-induced stratified density. Geophys. Astrophys. Fluid Dyn. (2020)

[14] Joseph, D.D.: Stability of Fluid Motions. Springer, Berlin (1976)

[15] Liu, X., Titi, E.S.: Well-posedness of strong solutions to the anelastic equations of stratified viscous flows. J. Math. Fluid Mech. 22(3), Paper no. 39 (2020) 
[16] Maremonti, P., Crispo, F., Grisanti, C.R.: Navier-Stokes equations: an analysis of a possible gap to achieve the energy equality. Ricerche di Matematica 70, 235-249 (2021)

[17] Masmoudi, N.: Rigorous derivation of the anelastic approximation. J. Math. Pures Appl. 88(3), 230-240 (2007)

[18] Passerini, A., Ruggeri, T.: The Bénard problem for quasi-thermal-incompressible materials: a linear analysis. Int. J. Nonlinear Mech. 67, 178-185 (2014)

[19] Prodi, G.: Teoremi di tipo locale per il sistema di Navier-Stokes e stabilità delle soluzioni stazionarie. Rend. del Sem. Matem della Univ. di Padova 3(2), 173-182 (1962)

[20] Rajagopal, K.R., Ruzicka, M., Srinivasa, A.R.: On the Oberbeck-Boussinesq approximation. Math. Mod. Meth. Appl. S. 6, 1157-1167 (1996)

[21] Rajagopal, K.R., Saccomandi, G., Vergori, L.: On the Oberbeck-Boussinesq approximation for fluids with pressure dependent viscosities. Nonlinear Anal. Real. 10, 1139-1150 (2009)

Arianna Passerini

Dipartimento di Matematica e Informatica

Università degli Studi di Ferrara

Via Machiavelli 30

44121 Ferrara

Italy

e-mail: arianna.passerini@unife.it

(accepted: August 22, 2021; published online: September 7, 2021) 